\title{
Interoperability is more than just technology
}

\author{
Bernd Blobel $^{1}$ and Mauro Giacomini ${ }^{2}$ \\ ${ }^{1}$ Medical Faculty, University of Regensburg, Germany \\ ${ }^{2}$ Dept. of Informatics, Bioengineering, Robotics and System Engineering, University of Genoa, Italy
}

\section{Correspondence to:}

Prof. Dr. habil. Bernd Blobel, FACMI, FACHI, FHL7

Medical Faculty, University of Regensburg

Address: c/o HL7 Deutschland e. V., An der Schanz 1, 50735 Köln

E-mail: bernd.blobel@klinik.uni-regensburg.de
EJBI 2016; 12(1):en1-en2

published: May 20, 2016
This Special Issue of the European Journal for Biomedical Informatics is dedicated to the International HL7 Interoperability Conference (IHIC 2016) "Interoperability is more than just technology", 13-15 June 2016 in Genoa, Italy [1]. It contains papers selected by an independent peer review process, strictly performed by experts from countries different from the authors' country of residence.

IHIC 2016 is the 16th event of the International HL7 Interoperability Conference series, which has been inaugurated in 2000 by the Board of HL7 Germany and its unforgettable Chair and interoperability pioneer Joachim W. Dudeck. The first event in Dresden, Germany, was entitled "Advanced Healthcare Information Standards". While the first conferences have been characterized by focusing on CDA (Clinical Document Architecture), over the time, the scope of the conferences has been extended towards all aspects of health information interoperability. The concept of interoperability has dramatically changed from standardized electronic data interchange (EDI) based on data representation at application level, the 7th level of the ISO Open Systems Interconnection stack, having been the name giver for the Health Level 7 standards framework. Meanwhile, the semantics of shared data as well as service level interoperability are considered, bringing terminology and ontology issues, but also implementation challenges such as Web services and RESTful technologies on board. As visible outcome of such development, requirements for National Interoperability Frameworks stated in the USA [2], but also hypes such as FHIR came up and are highlighted in the IHIC 2016 papers as well. So it is just consequent to address in 2016 also non-technological issues of interoperability.

The conference has been structured into four sections: a) Paradigm Changes in Healthcare and Resulting Interoperability Challenges, b) HL7 Perspectives, c) Terminology, Ontology and Classification Issues, and d) CDA-related Contributions. All sections have been introduced by related Keynotes. The papers published in this EJBI Special Issue address the different aspects of the interoperability challenge from a theoretical and methodological perspective, usability requirements, professional groups' preferences, process design, semantical ambiguity, and implementation details.
In his Keynote, Edward Hammond, Duke University (USA), discusses history as well as factors impacting the interoperability paradigm evolution, thereby especially referring to the situation in the United States. He claims use case specific interoperability definitions, and he investigates change management related obstacles in the evolution of practical interoperability. As plead over many years already, Bernd Blobel from the University of Regensburg (Germany) highlights in his Keynote that interoperability is not first at all an ICT challenge. Based on the definition of interoperability as comprehensive communication and cooperation of actors involved in health services to achieve common interests and business objectives, thereby considering their expectations and wishes in their specific environmental, social, and legal context, deploying their specific methodologies, knowledge, experiences, and skills, he introduces a Reference Architecture Model for abstracting, formalizing, and harmonizing real world business scenarios to realize advanced cross-domain, i.e. multi-disciplinary interoperability. In another Keynote, Riccardo Bellazzi, University of Pavia (Italy), approaches the advent of Big Data. Based on the outcome of EU funded projects, he explains how integration of data from heterogeneous sources can enable efficient decision support solutions.

Ken Salyards et al. present current standards and solutions for semantic interoperability of Electronic Health Record (EHR) systems. They propose a model driven approach to mapping different information representation formats, syntax and semantics. They offer Open Source solutions for components for a transformation/interface engine, thereby providing a clear separation of concerns between design and run-time throughout the development process. The ICT focused approach has been implemented at Substance Abuse and Mental Health Services Administration (SAMHSA) to manage cross-organizational behavioral and physical health care. Philip Scott, University of Portsmouth, UK, et al. present the innovative approach to EHR and EHR interoperability standards development led by end-users such as physicians with the support of patients instead of inaugurated by technicians, so extremely improving the acceptance of solutions. This process is managed by the Professional Record Standards Body (PRSB) for health and social 
care. The assessment and optimization of the process is performed using the normalization Process Theory. Contrary to the Salyards' article, this approach is closer related to objectives, methodologies and principles addressed in Blobel's Keynote. Alessandro Sulis and colleagues from the Center for Advanced Studies, Research and Development in Sardinia (Italy) present traceability based definition and management of clinical processes to respond to flexible data acquisition environments. For that purpose, the IHE LBL (Laboratory Specimen Barcode Labeling) profile has been used to model and practical implement a phlebotomy process. So, the authors could extend the IHE LBL profile by new transactions for containers production and samples collection. Mark Kramer, MITRE Corporation (USA), et al. use the profiling methodology to constrain Fast Healthcare Interoperability Resources (FHIR) for practical interoperability. In that context, criteria for compatibility, containment, and interoperability of FHIR profiles have been defined. The authors propose are more stringent process of developing FHIR core resources and profiles. Frank Oemig and Bernd Blobel try to predict the future of HL7's most successful and with several IHE Technical Frameworks supported interoperability standard: HL7 v2x. They offer a transition matrix to automatically generate new representations of the standard according to updated releases. The methodology offered is not limited to HL7 v2x, but also applicable to other specifications. Roberta Gazzarata and Mauro Giacomini, University of Genoa (Italy), present a standardized solution for clinical data sharing in acute care, telemedicine and clinical trials. For that purpose, they developed several basic services to be exploited by multiple systems in different care settings based on the SOA (Service Oriented Architecture) methodology and HL7's refinement to the Healthcare Service Specification Program, such as Health Record Management Services, Health Terminology Services and Health Identity Services. Humberto Fern $\breve{n}$ n Mandirola Brieux and colleagues from Argentina developed a clinical laboratory risk engine for automatically providing alerts on ICU patients, using HL7 2.6 specifications. They demonstrated the feasibility of their solution for risk factors blood glucose, hematocrit, WBCs, arterial blood gases, blood urea, blood creatinine, blood sodium and blood potassium. The assessment of the solution in comparison with a traditionally managed control group significantly demonstrated that clinical laboratory risks can be better detected when using an alert system. Abderrazek Boufahja and colleagues from IHE Europe present a tooling set for checking the conformance of specifications such as CDA implementation guides and CDA documents to implement HL7 specifications in IHE projects. The design of CDA documents such as HL7 Templates, supported by the ART-DÉCOR $R$ tool, can so be validated by formalizing the requirements with the Gazelle ObjectsChecker and transforming them correctly in IHE OCL constraints to enable practical interoperability. Stefan Sabutsch and Peter Seifter from HL7 Austria present a user centered design approach to CDA documents shared following the IHE Cross Enterprise Document Sharing specification (IHE XDS). For accommodating user preferences, they appropriately rendered HL7 CDA specs regarding structure, content, display style, etc. For that purpose, the usability of document design was assessed by analyzing the behavior of a test group. The authors recommend PDF/A-3 files including an attached CDA document as best practice for exchanging documents. Heike Dewenter and Sylvia Thun, University of Applied Sciences Niederrhein (Germany) tackle the problem of synonymous concepts for semantic interoperability in the context of the Von Willebrand disease, when using SNOMED-CT for terminology binding in HL7 V3 specifications. Elena Cardillo and coauthors from the NRC Institute of Informatics and Telematics, Cosenza (Italy), tackle the problem of semantic interoperability of EHR systems in a more general approach by presenting the Italian solution for managing and using medical terminologies and coding systems at national level. They emphasize the importance of standardized models and terminology services.

Additionally to the papers presented here, practice reports and implementation experiences have been shared at the conference. IHIC 2016 was completed by a Tutorial Day covering all topics connected to interoperability such as CDA, FHIR, SOA, but also security and privacy issues and the use of the HL7 InfoButton standard.

The Editors whish all interested parties an enjoyable reading.

The Guest-Editors are indebted to thank all authors and reviewers for their excellent work. Finally, they thank HL7 International and for sponsoring the event including the Joachim W. Dudeck Award as well as HL7 Czech Republic, HL7 Germany, HL7 Italy, and HL7 The Netherlands for financially supporting the Tutorials.

\section{References}

[1] http://ihic2016.eu

[2] Heath S. Interoperability under MACRA must provide standard HIE tools. EHR Intelligence, April 13, 2016 